\title{
TITLE:
}

\section{Theoretical study of Cl-related defect complexes in cubic SiC}

$\operatorname{AUTHOR}(S)$ :

Alfieri, G.; Kimoto, T.

\section{CITATION:}

Alfieri, G....[et al]. Theoretical study of Cl-related defect complexes in cubic SiC. Journal of Applied Physics 2012, 111(10): 103705.

ISSUE DATE:

2012-05-18

URL:

http://hdl.handle.net/2433/187959

\section{RIGHT:}

(C) 2012 American Institute of Physics. This article may be downloaded for personal use only. Any other use requires prior permission of the author and the American Institute of Physics. 


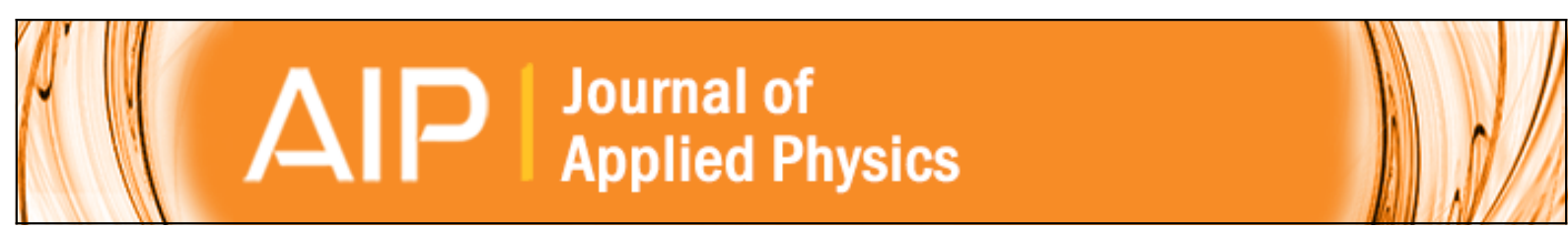

\section{Theoretical study of Cl-related defect complexes in cubic SiC}

G. Alfieri and T. Kimoto

Citation: Journal of Applied Physics 111, 103705 (2012); doi: 10.1063/1.4718030

View online: http://dx.doi.org/10.1063/1.4718030

View Table of Contents: http://scitation.aip.org/content/aip/journal/jap/111/10?ver=pdfcov

Published by the AIP Publishing

\section{Articles you may be interested in}

Study of properties of SiC layer in TRISO coated particles grown using different alkyl-silicon compounds

AIP Conf. Proc. 1538, 26 (2013); 10.1063/1.4810026

First-principles study of $\mathrm{Cl}$ diffusion in cubic SiC

J. Appl. Phys. 113, 133706 (2013); 10.1063/1.4799194

Electron paramagnetic resonance and theoretical studies of $\mathrm{Nb}$ in $4 \mathrm{H}$ - and $6 \mathrm{H}-\mathrm{SiC}$

J. Appl. Phys. 112, 083711 (2012); 10.1063/1.4759362

Defects in SiC for quantum computing

J. Appl. Phys. 109, 102417 (2011); 10.1063/1.3578264

Silicon vacancy in SiC: A high-spin state defect

Appl. Phys. Lett. 74, 221 (1999); 10.1063/1.123299

\section{AlP Re-register for Table of Content Alerts}




\title{
Theoretical study of Cl-related defect complexes in cubic SiC
}

\author{
G. Alfieri and T. Kimoto \\ Department of Electronic Science and Engineering, Kyoto University, Kyotodaigaku-katsura, Nishikyo, \\ Kyoto 615-8510, Japan
}

(Received 1 March 2012; accepted 16 April 2012; published online 18 May 2012)

\begin{abstract}
First-principles calculations were employed to investigate the electronic properties and formation energy of $\mathrm{Cl}$-related complex defects, with either carbon (silicon) vacancies, $V_{C}\left(V_{S i}\right)$ or dopants (nitrogen/aluminum). Our investigation revealed that $\mathrm{Cl}$ at a $\mathrm{Si}$-site related complexes, such as the $C l_{S i} V_{C}$ and $C l_{S i} N_{C}$, are either structurally unstable or possess a rather high formation energy, in agreement with the fact that $C l$ energetically favors a $C$-site. By employing the defect-molecule model, we found that $C l_{C} V_{S i}$ and $C l_{C} A l_{S i}$ complexes have $C_{3 v}$ symmetry and are both donors. However, contrarily to the case of the $C l_{C} V_{S i}$ complex, for the $C l_{C} A l_{S i}$ complex the singlet $\left(A_{1}\right)$ orbitals can lie higher in energy position than the doubly degenerate orbital $(E)$. The formation energies of $\mathrm{Cl}$-vacancy/dopant complex defects are discussed in the light of recent experimental results, reported for $4 \mathrm{H}-\mathrm{SiC}$ grown by $\mathrm{Cl}$-based chemical vapor deposition. (C) 2012 American Institute of Physics. [http://dx.doi.org/10.1063/1.4718030]
\end{abstract}

\section{INTRODUCTION}

Chlorine impurity plays an important role in group $I V$ semiconductors technology because $\mathrm{Cl}$ has turned out to be a suitable choice as dopant as in the case of silicon ${ }^{1}$ and diamond. ${ }^{2}$ For silicon, group $V$ dopants, such as $A s$, are subject to a high degree of deactivation, e.g., for an $A s$ concentration of $2 \times 10^{21} \mathrm{~cm}^{-3}$ only the $20 \%$ of the atoms are electrically active. ${ }^{1}$ The reason for the deactivation was attributed to the threefold coordination of these impurities which $\mathrm{Cl}$ is instead immune to, thus being an efficient shallow single donor. A similar issue also affects diamond for which $n$-type doping is still a major problem. While Pöykkö et al. ${ }^{3}$ reported that $\mathrm{Cl}$ behaves as a triple donor in diamond, but with states too deep in the band gap $\left(E_{G A P}\right)$ to promote sufficient electrons in the conduction band $\left(E_{C}\right)$, a more recent study ${ }^{2}$ has shown that $\mathrm{Cl}$ can be a good candidate for achieving $n$-type diamond.

Contrarily to silicon and diamond, $N$ and $A l$ are wellestablished dopants for $n-$ and $p$ - type SiC, respectively, but $\mathrm{Cl}$ has nevertheless revealed itself as a key element for $\mathrm{SiC}$ industry. In fact, $\mathrm{SiC}$ high-voltage power devices rely on very thick $(>30 \mu \mathrm{m})$ high-quality epilayers and while typical growth rates of $5-10 \mu \mathrm{m} / \mathrm{h}$ are employed for avoiding $\mathrm{Si}$-droplets formation ${ }^{4}$ during growth, they are inconvenient from an economic point of view. The use of chlorinated precursors in chemical vapor deposition (CVD) growth prevents $\mathrm{Si}$-droplets formation so that very high growth rates can be achieved (170 $\mu \mathrm{m} / \mathrm{h}$ (Ref. 5)). Cl-based CVD yields good quality epilayers, without stacking faults, ${ }^{6}$ that can be employed for $25 \mathrm{kV}$ blocking $\mathrm{SiC}$ devices.

Similarly to the case of $H$ impurities, ${ }^{7}$ it was shown that also $\mathrm{Cl}$ incorporation in $\mathrm{SiC}$, during growth, is feasible. $^{5,8}$ Yet, even if $\mathrm{Cl}$ concentration was reported to be below the detection limit $\left(\sim 10^{14} \mathrm{~cm}^{-3}\right),{ }^{5}$ the possibility that $\mathrm{Cl}$ atoms are incorporated in the $10^{11}-10^{12} \mathrm{~cm}^{-3}$ range, cannot be excluded. This has raised the question on whether or not, $\mathrm{Cl}$ gives rise to electrically active defects in the band gap but, despite the technological importance of this matter, only recently it was found that both substitutional and interstitial $\mathrm{Cl}$ give rise to electrically active levels in $E_{G A P}$. In addition, substitutional $C l$ behaves as a triple donor in $\mathrm{SiC},{ }^{9}$ consistently with the fact that $N$ and $O$ are a single ${ }^{10}$ and double ${ }^{11}$ donor, respectively. However, the behavior of $\mathrm{Cl}$ in the presence of vacancies, e.g., in irradiated/implanted epilayers, as well as its interaction with $n-$ or $p$ - type doping, is not known, yet. For instance, both $n$ - and $p$ - type dopants can be achieved by $\mathrm{Cl}$-based CVD but it is not clear why, while no significant effect on $\mathrm{N}$ incorporation was reported for increasing $\mathrm{N}_{2} / \mathrm{Si} \mathrm{ratio}^{12}$, Al concentration reaches saturation at $\sim 10^{18} \mathrm{~cm}^{-3}$ for increasing $\mathrm{Al} / \mathrm{Si}$ ratio. $^{13}$

For this reason, in order to provide an explanation for this experimental findings and provide a more detailed picture of the behavior of $\mathrm{Cl}$ impurity in $\mathrm{SiC}$, we report on a first-principles study of the geometry and electronic properties of substitutional $\mathrm{Cl}$-related defect complexes, with either carbon (silicon) vacancies, $V_{C}\left(V_{S i}\right)$, or dopant impurities $(A l, N)$.

\section{COMPUTATIONAL METHOD}

Spin-polarized density functional calculations of the equilibrium configurations and formation energies $E_{f o r m}$ of $\mathrm{Cl}$-related defect complexes were carried out on a cubic $\mathrm{SiC}$ supercell consisting of 64 atoms. We employed the Siesta package,${ }^{14}$ in the local density approximation (LDA) with the exchange correlation functional of Ceperley and Alder ${ }^{15}$ and norm-conserving Troullier-Martins pseudopotentials ${ }^{16}$ and a double- $\zeta$ plus polarization atomic orbitals basis set. Further details and convergence tests can be found elsewhere. ${ }^{9}$

The formation energy, $E_{\text {form }}$, employed to calculate the position of the transition levels in the Kohn-Sham band gap (estimated by employing the Madelung correction), $E_{G A P}$, was obtained by using Eq. (17) of Ref. 17 


$$
\begin{aligned}
E_{\text {form }}^{q}= & E_{\text {tot }}^{q}-\frac{n_{S i}+n_{C}}{2} \mu_{S i C}^{b u l k}-\frac{n_{S i}-n_{C}}{2}\left(\mu_{S i}^{b u l k}-\mu_{C}^{b u l k}\right) \\
& -\left(n_{S i}-n_{C}\right) \Delta \mu-n_{C l} \mu_{C l}+q E_{F}
\end{aligned}
$$

with $q, E_{t o t}, n_{S i, C, C l}, \mu_{S i, C, S i C}^{b u l k}, \mu_{C l}, E_{F}$ being the charge state, the total energy of the supercell containing the defect, the number of $\mathrm{Si}, \mathrm{C}, \mathrm{Cl}$ atom, the total energy per atom of $\mathrm{Si}$ and $C$ in the diamond structure, the total energy per $\mathrm{Si}-\mathrm{C}$ pair in bulk $\mathrm{SiC}$, the chemical potential of $\mathrm{Cl}$ and the Fermi energy, respectively. The chemical potentials of $S i$ and $C$ are connected by $\mu_{S i C}^{\text {bulk }}=\mu_{S i}+\mu_{C}$ and introducing $\Delta \mu_{S i, C}=\mu_{S i, C}$ $-\mu_{S i, C}^{b u l k}$ and the formation enthalpy $\Delta H_{f}^{S i C}=\mu_{S i C}^{b u l k}-\left(\mu_{S i}^{b u l k}\right.$ $\left.+\mu_{C}^{b u l k}\right)$, we can define $\Delta \mu=\Delta \mu_{S i}-\frac{1}{2} \Delta H_{f}^{S i C}=\frac{1}{2} \Delta H_{f}^{S i C}$ $-\Delta \mu_{C}$. It follows that it is possible to distinguish between $\mathrm{C}$-rich and Si-rich conditions by

$$
\frac{1}{2} \Delta H_{f}^{S i C} \leq \Delta \mu \leq-\frac{1}{2} \Delta H_{f}^{S i C}
$$

In the following, we will limit our discussion to the stoichiometric limit $(\Delta \mu=0)$.

Regarding the chemical potential of $C l(N)$, it was calculated for the $\mathrm{Cl}_{2}\left(N_{2}\right)$ molecule at the temperature of $1570{ }^{\circ} \mathrm{C}$ and pressure of 200 mbar. $^{5} \mu_{C l}\left(\mu_{N}\right)$ is then obtained from the following equation ${ }^{18}$

$$
2 \mu_{C l}=E_{t o t}\left(C l_{2}\right)+\tau \ln (p V / \tau)-\tau \ln \Theta_{r o t}-\tau \ln \Theta_{v i b}
$$

with $E_{\text {tot }}$ the total energy of the $C l_{2}\left(N_{2}\right)$ molecule, $p$ the pressure, $V=\left(h^{2} / 2 \pi m k T\right)^{3} / 2, k_{B}$ the Boltzmann constant, $T$ the temperature, $\tau=k_{B} T$ and $\Theta_{\text {rot,vib }}$ (Ref. 19) are the rotational and vibrational contributions to the partition function.

In order to test the validity of our method, we calculated the energy position in the $E_{G A P}$ of the triply degenerate Kohn-Sham level of the silicon vacancy $V_{S i}\left(T_{d}\right)$ and that of the $A l_{S i}$ singly negative charge state, by employing the $E_{\text {form }}$. In the former case, we found that the partially occupied triply degenerate level $\left(T_{2}\right)$ is located at $0.47 \mathrm{eV}$ above the maximum of the valence band, $E_{V}$, in agreement with previous reports. ${ }^{20}$ In the latter, the calculated $E_{\text {form }}$ for $A l_{S i}$ is negative $(-3.24 \mathrm{eV})$, analogously to the results of Fukumoto, ${ }^{10}$ and the acceptor level is $0.23 \mathrm{eV}$ above $E_{V}$, in fair agreement with the experimental values reported in the literature. ${ }^{21}$

\section{RESULTS AND DISCUSSION}

In Fig. 1, the $\mathrm{Cl}$-related defect complexes, examined in this study, are shown. For the $C l_{C} V_{S i}$ complex (Fig. 1(a)), after geometry optimization, similarly to the case of $\mathrm{Cl}_{C}{ }^{9}$, the $\mathrm{Si}$ first-neighbors relax outwards and the $\mathrm{Cl}-\mathrm{Si}$ bond length increases by $0.05 \AA$. However, while for $C l_{C}$, the $C l$ did not move away from its position in the $V_{C}$, for the $C l_{C} V_{S i}$ complex, the $C l$ atom moves slightly toward the $V_{S i}$. In Fig. 1(b), the $C l_{S i} V_{C}$ complex is shown in its unrelaxed position and it is interesting to note that after geometry optimization, the $\mathrm{Cl}$ atom moves at $2.88 \AA$ away from its $C$ first-neighbors, leaving the $V_{S i}$, and ending up in the $V_{C}$ at $2.31 \AA$ from the $S i$ atoms. The explanation of the transformation of the $C l_{S i} V_{C}$ complex into $\mathrm{Cl}_{C} V_{S i}$ can be found in the fact that $C l$ atoms
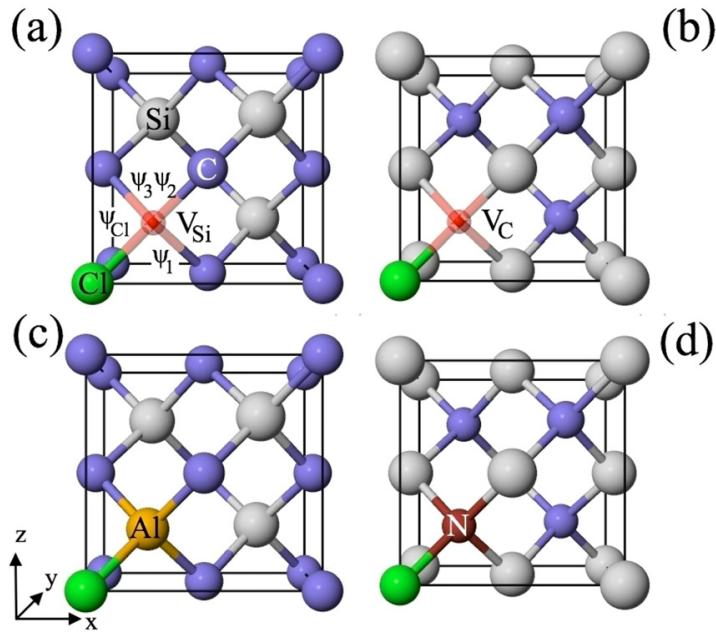

(d)

FIG. 1. The unrelaxed structure of the four investigated $\mathrm{Cl}$-related complex defects. (a) $C l_{C} V_{S i}$, (b) $C l_{S i} V_{C}$, (c) $C l_{C} A l_{S i}$, and (d) $C l_{S i} N_{C}$. Carbon atoms are blue, silicon is light grey, chlorine is green, aluminum is orange, and nitrogen is brown. The translucent red ball represents a vacancy (carbon or silicon).

are not stable in a $S i$-site but they energetically favor a $C$-site. ${ }^{9}$ In Figs. 1(c) and 1(d), the $C l_{C} V_{S i}$ and $C l_{S i} N_{C}$ are displayed, respectively. For the relaxed structure of the $C l_{S i} N_{C}$ complex, contrarily to the case of $C l_{C} A l_{S i}$ for which the $C l$ atom remains in the $V_{C}$ and the $A l$ atom moves slightly off $(0.3 \AA)$ the $V_{S i}$, the $C l$, and the $N$ atoms move away from each other and the $\mathrm{Cl}-\mathrm{N}$ bond is $\sim 1 \AA$ longer than the one found in the $\mathrm{ClN}_{3}$ molecule. ${ }^{22}$ This behavior can be explained in terms of Coulombic repulsion because, in $\mathrm{SiC}$, both $\mathrm{N}$ and $\mathrm{Cl}$ atoms impurities have a donor nature.

In Fig. 2, the total electron density, obtained for the relaxed structures, is presented. It can be seen that, in general, the charge density is mainly localized on $\mathrm{C}, \mathrm{N}$, and $\mathrm{Cl}$ atoms, due to their high electronegativity. For instance, in Fig. 2(a), for $C l_{C} V_{S i}$ (and $C l_{S i} V_{C}$, too) the $C l_{C}-V_{S i}$ bond has an ionic character, as the charge density is mainly localized on the $\mathrm{Cl}$ atom. The same can be said for the $C l_{C} A l_{S i}$ complex (Fig. 2(b)), even if, as it can also be noted, charge transfer is present between the $C l$ atom and the $C$ first-neighbor of the $A l$ atom and between the $C l$ and $A l$ atoms. For the $\mathrm{N}-\mathrm{Cl}$ bond, shown in Fig. 2(c), the electrons are mainly localized on the $\mathrm{N}$ and $\mathrm{Cl}$ atoms, however, some charge is present halfway along the bond direction, indicating a weak covalent bond.

By employing the defect-molecule model, we analyzed the electronic structure of the investigated complexes. We will focus our discussion on the $C l_{C} V_{S i}$ and $C l_{C} A l_{S i}$ complexes due to the higher stability of $\mathrm{Cl}_{C}$ compared to $C l_{S i}$. Regarding the $C l_{C} V_{S i}$ complex (Fig. 1(a)), we follow a similar analysis developed for the $N_{C} V_{C}$ complex in diamond, ${ }^{23}$ with the vacancy ( $A l$ atom) placed in the center of a tetrahedron at the corners of which are placed three $\mathrm{C}$ atoms and the $C l$ impurity. The total number of electrons in the $C l_{C} V_{S i}$ system is seven: the four electrons of the dangling bonds of the $V_{S i}$ plus the three electrons of the $C l$ impurity which is a triple donor. The molecular orbitals, in which the electrons will be accommodated, can be constructed by linear 

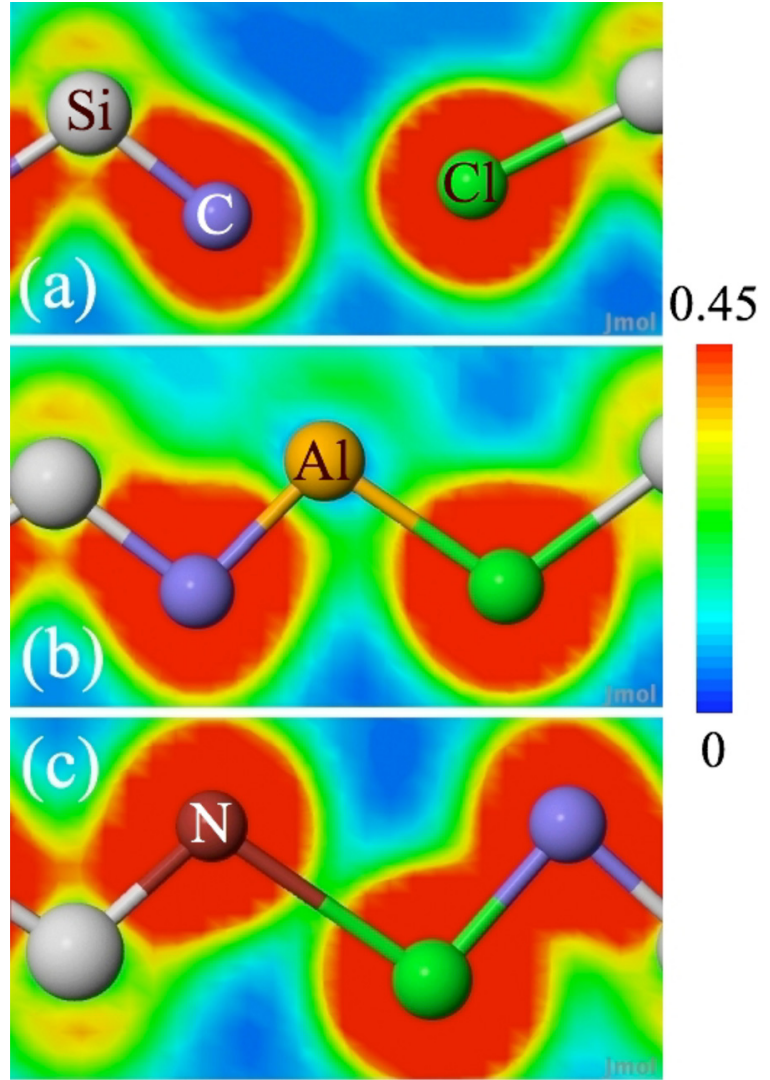

0

FIG. 2. Total electron density map for the relaxed structures of the (a) $C l_{C} V_{S i}\left(C l_{S i} V_{C}\right)$, (b) $C l_{C} A l_{S i}$, and (c) $C l_{S i} N_{C}$ defects. The units are expressed in $e^{-} / \AA^{3}$.

combination of atomic orbitals, labeled $\psi_{C l}, \psi_{1}, \psi_{2}$, and $\psi_{3}$ and by using the projector operator technique. ${ }^{24}$ In the present case, for the $C_{3 v}$ symmetry, we have

$$
\begin{gathered}
a_{1}(1)=\psi_{C l} \\
a_{1}(2)=\left(\psi_{1}+\psi_{2}+\psi_{3}-3 \lambda \psi_{C l}\right) / \sqrt{3} S_{1} \\
e_{x}=\left(2 \psi_{3}-\psi_{1}-\psi_{2}\right) / \sqrt{3} S_{2} \\
e_{y}=\left(\psi_{1}-\psi_{2}\right) / S_{2}
\end{gathered}
$$

with the following overlap integrals $S_{1}=\sqrt{1+2 \mu-3 \lambda^{2}}$, $S_{2}=\sqrt{2-2 \mu}$ with $\lambda=\left\langle\psi_{C l} \mid \psi_{1}\right\rangle$ and $\mu=\left\langle\psi_{1} \mid \psi_{2}\right\rangle$. Following Fig. 2(a), we neglect the overlap terms and set $\lambda=\mu=0$. In this way, the energies of the molecular orbitals become:

$$
\begin{gathered}
U_{1}=\left\langle a_{1}(1)|H| a_{1}(1)\right\rangle \approx \alpha_{0} \\
U_{2}=\left\langle a_{1}(2)|H| a_{1}(2)\right\rangle \approx \alpha+2 \beta \\
U_{x(y)}=\left\langle e_{x(y)}|H| e_{x(y)}\right\rangle \approx \alpha-\beta,
\end{gathered}
$$

where the charge transfer terms are defined as $\alpha=\left\langle\psi_{i}|H| \psi_{i}\right\rangle$ $(\mathrm{I}=1,2,3), \quad \beta=\left\langle\psi_{i}|H| \psi_{j}\right\rangle \quad(\mathrm{I}=1,2,3, \quad \mathrm{i} \neq \mathrm{j}), \quad$ and $\quad \alpha_{0}$ $=\left\langle\psi_{C l}|H| \psi_{C l}\right\rangle$. Being $V_{S i}$ a cation vacancy, $\beta<0,{ }^{25}$ while $\alpha>\alpha_{0}\left(\alpha_{0}<0\right)$ due to the higher electronegativity of $C l$ than that of $C$ atoms. We then obtain that $U_{x(y)}$ lies higher in energy than $U_{1}$ and $U_{2}\left(U_{x(y)}>U_{2}>U_{1}\right)$, provided that $\alpha_{0}<\alpha+2 \beta$.

For the case of the $\mathrm{Cl}_{C} A l_{S i}$ complex, having a $C_{3 v}$ symmetry, the above linear combination of atomic orbitals is still valid. However, there are now ten electrons (four of the $V_{S i}$, the three valence electrons of $\mathrm{Al}$ and the three of $\mathrm{Cl}$ donor) to accommodate in the molecular orbitals. As shown in Fig. 2(b), a certain degree of interaction is present between the $\mathrm{Cl}$ atom and the $\mathrm{Al}$ atom first neighbors, thus we can set $\lambda \neq 0$ and introduce a new transfer term $\gamma=\left\langle\psi_{1}|H| \psi_{C l}\right\rangle$. Equations (5) now become

$$
\begin{gathered}
U_{1}=\left\langle a_{1}(1)|H| a_{1}(1)\right\rangle \approx \alpha_{0} \\
U_{2}=\left\langle a_{1}(2)|H| a_{1}(2)\right\rangle \approx\left(\alpha+2 \beta+3 \lambda^{2} \alpha_{0}-2 \lambda \gamma\right) /\left(1-3 \lambda^{2}\right) \\
U_{x(y)}=\left\langle e_{x(y)}|H| e_{x(y)}\right\rangle \approx \alpha-\beta
\end{gathered}
$$

with $U_{1}$ being the lowest in energy. Regarding $U_{2}$, if $\lambda=0$, the molecular orbitals follow the same energy order as described above, while for $\lambda=1$ both the denominator and numerator become negative so that $U_{x(y)}$ and $U_{2}$ switch positions, provided that $\alpha_{0}>\frac{2}{3} \gamma-\alpha$.

In order to verify if the energy sequence of the molecular orbitals, as predicted by group theory is correct, we show the molecular orbitals obtained by our density functional calculations (Fig. 3). For the $C l_{C} V_{S i}$ complex, two of the total seven electrons occupy a singlet $a_{1}(1)$ orbital, resonant in $E_{V}$ (Fig. 3(a)); The next two electrons can be found in another singlet $a_{1}(2)$ orbital (Fig. 3(b)), located at $0.18 \mathrm{eV}$ above $E_{V}$, other two occupy an antibonding $a_{1}(1)$ level (Fig. 3(c)),

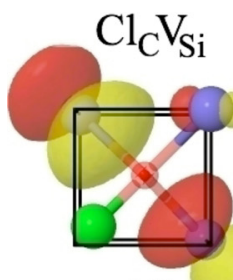

(d)

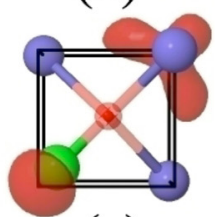

(c)

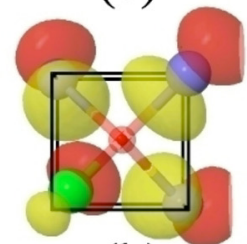

(b)

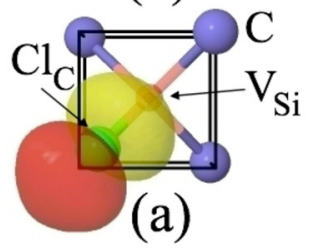

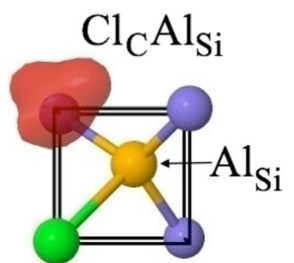

(h)

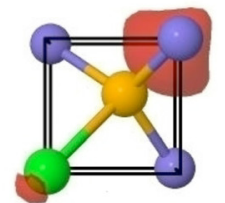

(g)

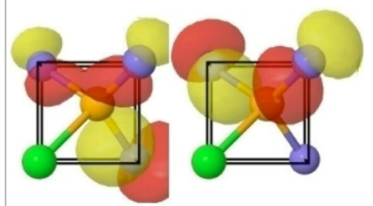

(f)

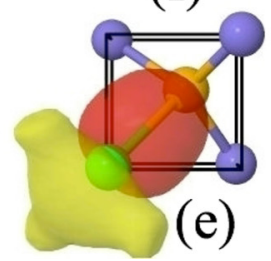

FIG. 3. Isosurfaces of the calculated wave functions of the defect states $\left(0.095 e^{-} / \AA^{3}\right)$. Red (yellow) isosurfaces represent the positive (negative) values of the wave function. On the left column, the isosurfaces of the (a) $a_{1}(1)$, (b) $a_{1}(2)$, (c) $a_{1}(1)^{*}$, and (d) $e_{x}$ for the $C l_{C} V_{S i}$ complex, are shown. On the right, the (e) $a_{1}(1)$, (f) $e_{x}, e_{y},(\mathrm{~g}) a_{1}(1)^{*}$, and (h) minimum of the conduction band, for the $C l_{C} A l_{S i}$ defect are presented. 


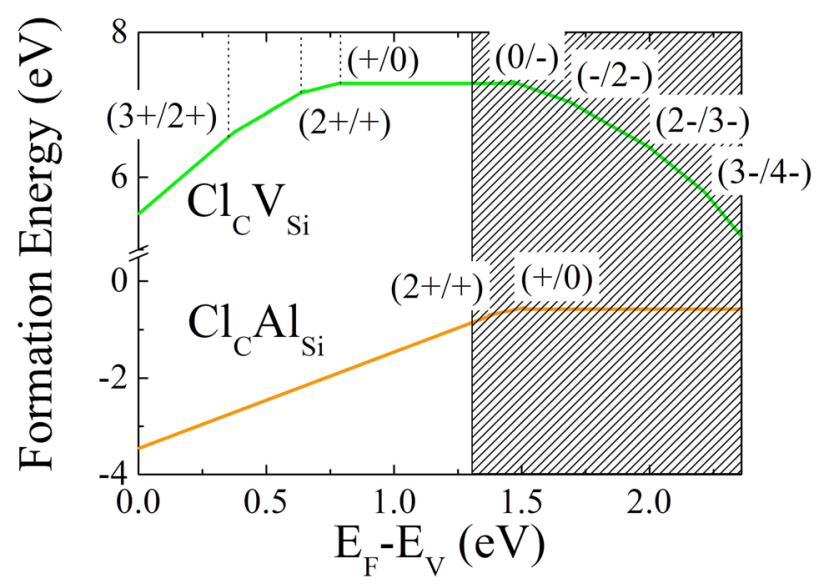

FIG. 4. Formation energies of the $C l_{C} V_{S i}$ and $C l_{C} A l_{S i}$ complex defects, after geometry relaxation. The Madelung correction was used to calculate the formation energy of charged defects and the shadowed area delimits the KohnSham band gap.

corresponding to a Kohn-Sham state at $0.86 \mathrm{eV}$ above $E_{V}$, while the last electron is found in a doubly degenerate orbital $e_{x}$ (Fig. 3(d)), at $1.02 \mathrm{eV}$ above $E_{V}$.

For the $C l_{C} A l_{S i}$ complex, density functional calculations show that the molecular orbitals follow the energy pattern described for the $\lambda=1$ case. In fact, two fully occupied levels are found, resonant in the valence band, one corresponding to the $a_{1}(1)$ orbital (Fig. 3(e)) and another one to the doubly degenerate $e_{x}, e_{y}$ (Fig. 3(f)) levels. Of the four remaining electrons, two are found in a singly occupied Kohn-Sham state at $0.57 \mathrm{eV}$ above $E_{V}$, corresponding to an antibonding $a_{1}(1)$ orbital (Fig. 3(g)), while the other two are resonant in the conduction band (at $\sim 1.5 \mathrm{eV}$ above the minimum of the conduction band, $E_{C}$ ) therefore they are transferred to $E_{C}$ (Fig. 3(h)).

We next analyzed the $E_{\text {form }}$ of the studied complexes, as a function of the Fermi energy $\left(E_{F}\right)$ (Fig. 4). The formation of the $C_{C} V_{S i}$ complex can occur after particle irradiation/implantation of chlorinated $\mathrm{SiC}$ epilayers, or after $\mathrm{Cl}$ implantation, when vacancies are trapped by $C l$ impurities. The $E_{\text {form }}$ of $C l_{C} V_{S i}$, as well as that of the $C l_{S i} V_{C}$ complex, is rather high $(\sim 7.3 \mathrm{eV})$ and for this reason, this defect should be present in negligible concentrations, but it may be more likely to form in either strongly $n-$ or $p$ - type material. If we focus on the Kohn-Sham $E_{G A P}$ (delimited by the shadowed area of Fig. 4), for $n$-type conditions, the $C l_{C} V_{S i}$ complex can be found in the neutral charge state while for slightly $n$-type and $p$-type material, three donor levels can be found in $E_{G A P}$, at 0.35 , 0.64 , and $0.78 \mathrm{eV}$ above $E_{V}$, corresponding to the triple, double, and single charge states. Four negative charge states can be found resonant in the Kohn-Sham $E_{C}$, all stable within small ranges of $E_{F}$. To date, no reports on the presence of chlorine-vacancy complexes can be found in the literature. $\mathrm{La}$ Via et $a{ }^{4}{ }^{4}$ have shown that in $n$-type $4 \mathrm{H}-\mathrm{SiC}$ grown by $\mathrm{Cl}$-based $\mathrm{CVD}$, only the $\mathrm{Z}_{1 / 2}$ and $\mathrm{EH}_{6 / 7}$ are present, meaning that $\mathrm{Cl}$ precursors should not give rise to new deep levels in $E_{G A P}$. Yet, the study was performed on as-grown samples and it can be argued that particle irradiation or ion-implantation may generate a high enough vacancy concentration so that $C l_{C} V_{S i}$ complexes can be formed. Moreover, La Via et al. ${ }^{4}$ have performed their study on epitaxial layers grown at $1600{ }^{\circ} \mathrm{C}$, a similar temperature to the one considered in the present study, which is responsible for the high $E_{\text {form }}$ of the $C l_{C} V_{S i}$ defects. If lower growth temperatures were to be used, lower $E_{\text {form }}$ would be needed for $C l_{C} V_{S i}$ to be present in the epilayers, as it can be deduced by Eq. (3).

Regarding the $C l_{S i} N_{C}$ complex, this has a higher $E_{\text {form }}$ energy than $C l_{C} A l_{S i}$ due to the instability of $C l$ in a $S i$-site and coulombic repulsion. For this reason, the formation of a $N$-related complex should be unlikely. As Pedersen et al. ${ }^{12}$ have reported, the net-donor concentration $\left(N_{d}\right)$ is not affected by the use of chlorinated precursors during growth. The reported decrease of $N_{d}$, for increasing $C / S i$ ratio, can instead be related to the decrease of available vacant $\mathrm{C}$-sites for the $N$ impurity rather than to the presence of $\mathrm{Cl}$. In fact, even if $C l_{C}$ is energetically favored with respect to $C l_{s i}$, its $E_{\text {form }}$ is much higher than that of $N_{C}$.

Contrarily to the case of $C l_{S i} N_{C}$, the $E_{\text {form }}$ of $C l_{C} A l_{S i}$ is rather low (Fig. 4) meaning that it should form spontaneously and possess a rather high thermal stability. In fact, chlorinated aluminum species, such as $\mathrm{AlCl}$ are stable up to $2200{ }^{\circ} \mathrm{C}$. ${ }^{12} \mathrm{As}$ previously anticipated, the $C l_{C} A l_{S i}$ is a donor and the doubly charged state is stable for all the values of $E_{F}$ in the KohnSham $E_{G A P}$ (delimited by the shadowed are in Fig. (4)). This means that $C l_{C} A l_{S i}$ complex should not affect $N_{d}$ in $n$-type material (although the presence of $A l$ impurity would be highly unlikely in that case) but may have important consequences in intentionally $A l$-doped $\mathrm{SiC}$ epilayers grown by $C l$-based CVD. In fact, as $A l$ dopants are incorporated in the epilayers, the formation of $C l_{C} A l_{S i}$ complex defects is favored over that of the isolated $C l_{C} \cdot{ }^{9}$ Since the $E_{\text {form }}$ of $A l_{S i}$ is lower than that of $C l_{C} A l_{S i}$, over a wide range of $E_{F}$ values, $p$-type doping is achievable in $C l$-based CVD grown $\mathrm{SiC}$. However, when $E_{F}=E_{V}+0.08 \mathrm{eV}, C l_{C} A l_{S i}$ and $A l_{S i}$ have the same $E_{\text {form }}$ meaning that donors $\left(C l_{C} A l_{S i}\right)$ can now compensate the acceptors $\left(A l_{S i}\right)$. This can explain the saturation of $N_{a}$ for increasing $\mathrm{Al} / \mathrm{Si}$ ratio, that was reported by Pedersen et al., ${ }^{13}$ in $\mathrm{Al}$-doped $4 \mathrm{H}$-SiC epilayers grown by Cl-based CVD.

\section{CONCLUSIONS}

The electronic properties of four $\mathrm{Cl}$-related complex defects were investigated by means of ab-initio calculations. The study of the calculated molecular orbitals confirmed the preliminary results of our group theoretical analysis. In fact, it was found that, while the molecular orbitals of the cationvacancy related defect follow the usual pattern with a doubly degenerate orbital higher in energy than singlet ones, for a $C l_{C} A l_{S i}$ defect the doubly degenerate orbital can occupy a lower energy position than that of singlet orbitals. Furthermore, it was shown that, unlike $C l_{S i} N_{C}$ complex defect, $C l_{C} A l_{S i}$ can form spontaneously in intentionally $A l$-doped $\mathrm{SiC}$ grown by $\mathrm{Cl}$-based $\mathrm{CVD}$ and may lead to net acceptor compensation.

\section{ACKNOWLEDGMENTS}

This work was supported by the Japan Society for the Promotion of Science and by the Global COE Program 
(C09) from the Ministry of Education, Culture, Sports and Technology, Japan.

${ }^{1}$ D. J. Chadi, Appl. Phys. Lett. 71, 806 (1997).

${ }^{2}$ C. X. Yan, Y. Dai, and B. B. Huang, J. Phys. D: Appl. Phys. 42, 145407 (2009). ${ }^{3}$ S. Pöykkö, M. Kaukonen, M. J. Puska, and R. M. Nieminen, Comput. Mater. Sci. 10, 351 (1998).

${ }^{4}$ F. La Via, G. Galvagno, G. Foti, M. Mauceri, S. Leone, G. Pistone, G. Abbondanza, A. Veneroni, M. Masi, G. L. Valente, and D. Crippa, Chem. Vap. Deposition 12, 509 (2006).

${ }^{5}$ H. Pedersen, S. Leone, A. Henry, V. Darakchieva, P. Carlsson, A. Gällström, and E. Janzén, Phys. Status Solidi (RRL) 2, 188 (2008).

${ }^{6}$ S. Leone, Y. C. Lin, F. C. Beyer, S. Andersson, H. Pedersen, O. Kordina, A. Henry, and E. Janzén, Mater. Sci. Forum 679-680, 59 (2011).

${ }^{7}$ N. Achtziger, J. Grillenberger, W. Witthuhn, M. K. Linnarsson, M. Janson, and B. G. Svensson, Appl. Phys. Lett. 73, 945 (1998).

${ }^{8}$ B. Reznik, D. Gerthsen, W. Zhang, and K. J. Hüttinger, J. Eur. Ceram. Soc. 23, 1499 (2003).

${ }^{9}$ G. Alfieri and T. Kimoto, J. Phys.: Condens. Matter 23, 415802 (2011).

${ }^{10}$ A. Fukumoto, Phys. Status Solidi B 202, 125 (1997).

${ }^{11}$ A. Gali, D. Heringer, P. Deák, Z. Hajnal, Th. Frauenheim, R. P. Devaty, and W. J. Choyke, Phys. Rev. B 66, 125208 (2002).
${ }^{12}$ H. Pedersen, F. C. Beyer, J. Hassan, A. Henry, and E. Janzén, J. Cryst. Growth 311, 1321 (2009).

${ }^{13}$ H. Pedersen, F. C. Beyer, A. Henry, and E. Janzén, J. Cryst. Growth 311, 3364 (2009).

${ }^{14}$ J. M. Soler, J. D. Gale, A. Garcia, J. Junquera, P. Ordejón, and D. Sanchez-Portal, J. Phys.: Condens. Matter 14, 2745 (2002).

${ }^{15}$ D. M. Ceperley and B. J. Alder, Phys. Rev. Lett. 45, 566 (1980).

${ }^{16}$ N. Troullier and J. L. Martins, Phys. Rev. B 43, 1993 (1991).

${ }^{17}$ B. Aradi, A. Gali, P. Deák, J. E. Lowther, N. T. Son, E. Janzén, and W. J. Choyke, Phys. Rev. B. 63, 245202 (2001).

${ }^{18}$ J. E. Northrup, R. Di Felice, and J. Neuebauer, Phys. Rev. B 56, R4325 (1997).

${ }^{19}$ T. L. Hill, An Introduction to Statistical Mechanics (Dover, New York, 1986).

${ }^{20}$ A. Zywietz, J. Furthmüller, and F. Bechstedt, Phys. Rev. B. 59, 15166 (1999).

${ }^{21}$ G. Pensl and W. J. Choyke, Physica B 185, 264 (1993).

${ }^{22}$ R. L. Cook and M. C. L. Gerry, J. Chem. Phys. 53, 2525 (1970).

${ }^{23}$ A. Lenef and S. C. Rand, Phys. Rev. B 53, 13441 (1996).

${ }^{24}$ M. Tinkham, Group Theory and Quantum Mechanics (Dover, New York, 1992).

${ }^{25}$ T. Chanier, I. Opahle, M. Sargolzaei, R. Hayn, and M. Lannoo, Phys. Rev. Lett. 100, 026405 (2008). 